\title{
Predictive value of procalcitonin in chronic allograft dysfunction in kidney transplant recipients
}

\author{
JING YAO $^{1}$, LIJUAN JIANG ${ }^{2}$, DONG XUE ${ }^{3}$ and YANBEI SUN ${ }^{4}$ \\ ${ }^{1}$ Blood Purification Centre, The Affiliated Changzhou No. 2 People's Hospital of Nanjing Medical University; \\ Departments of ${ }^{2}$ Clinical Laboratory, ${ }^{3}$ Urology and ${ }^{4}$ Nephrology, The Third Affiliated Hospital of Soochow University, \\ Changzhou, Jiangsu 213003, P.R. China
}

Received February 22, 2019; Accepted September 12, 2019

DOI: $10.3892 / \mathrm{etm} .2019 .8113$

\begin{abstract}
The present study was designed to determine the potential role of circulating procalcitonin (PCT) in predicting chronic allograft dysfunction (CAD) in kidney transplant recipients (KTRs). A total of 87 KTRs were retrospectively analyzed and divided into a CAD and a non-CAD (normal renal function) group. Clinical features and inflammatory markers were compared between the groups, including PCT, white blood cell count, C-reactive protein, neutrophil percentage (N\%) and lipoprotein(a) [Lp(a)], and the receiver operating characteristic (ROC) curve for CAD prediction was plotted. Univariate and multivariate logistic regression analyses were used to analyze the relevant risk factors for CAD. The results indicated that i) the values of these indicators in the CAD group, including the male ratio, years after transplantation, PCT, N\% and Lp(a), were significantly higher than those in the non-CAD group, while the body mass index, aspartate aminotransferase, high-density lipoprotein and low-density lipoprotein were significantly lower; ii) PCT and $\mathrm{Lp}(\mathrm{a})$ were able to predict $\mathrm{CAD}$ with an area under the ROC curve of 0.893 and 0.770 , respectively; iii) multivariate logistic regression analysis of factors influencing CAD in KTRs suggested that elevated PCT was an independent risk factor. In KTRs, PCT was identified as a potential biomarker for predicting CAD.
\end{abstract}

\section{Introduction}

All over the world, 40,000 organ transplants are performed per annum, among which kidney transplants are the most common procedure (1); however, most of the transplanted kidneys gradually develop functional failure within

Correspondence to: Dr Yanbei Sun, Department of Nephrology, The Third Affiliated Hospital of Soochow University, 185 Juqian Street, Changzhou, Jiangsu 213003, P.R. China

E-mail: sunyb-1985@163.com

Key words: procalcitonin, chronic allograft dysfunction, kidney transplant recipients, low-grade inflammation
10 years (2). In solid organ transplantation, chronic low-grade inflammation is thought to participate in chronic allograft dysfunction (CAD), the leading cause of late renal allograft dysfunction (3). Atherosclerosis, as one of the conditions associated with chronic low-grade inflammation, promotes the progression of CAD (4).

In 1993, the potential of circulating procalcitonin (PCT) as a biomarker for severe systemic inflammation, infection and sepsis was first suggested (5). Later studies have indicated that elevated PCT may be observed in patients with chronic kidney disease (CKD), regardless of the presence of any infection $(6,7)$. A previous study identified a negative association between the baseline value of PCT and renal function in CKD patients (8). Low-grade inflammatory responses or a micro-inflammatory state in CKD patients are thought to cause an increase in pro-inflammatory metabolites and stimulation of the immune system, leading to increased release of inflammatory mediators and PCT entering the blood circulation. van Ree et al (4) reported that $\mathrm{PCT}$ may reflect chronic low-grade inflammation in a non-infected state that persists in the transplanted kidneys. In their study, the endpoint of the renal event was graft failure, return to dialysis, retransplantation or death, while mild to moderate-stage CAD was not among the items assessed. In addition, the levels of PCT after the acute phase of infection were not followed up.

Besides creatinine clearance and proteinuria, certain biomarkers are available to predict mild to moderate CAD of KTRs and clinicians are exploring novel non-invasive methods for entirely or partially replacing renal allograft biopsy for diagnosis of CAD. The aim of the present study was to assess the potential predictive value of circulating PCT concentrations for CAD in KTRs. Due to the high prevalence of infection in the KTRs who received long-term immunosuppressive agents compared with that in healthy subjects, patients with severe infections were therefore excluded. in the present study.

\section{Materials and methods}

General information. The present study was approved by the Ethics Committee of The Third Affiliated Hospital of Soochow University (Changzhou, China). A total of 92 KTRs from the Department of Nephrology, the Third Affiliated Hospital of Soochow University in Changzhou, P.R. China, 
were followed up after kidney transplant between March 2014 and September 2017, by collecting the data retrospectively. All medical records were anonymous and all patients provided written informed consent. The subjects that met the inclusion criteria were adults ( $>18$ years of age) who had undergone kidney transplantation more than a year prior to their inclusion in this present study. Patients who had undergone major surgery in the past 5 years or had severe cardiogenic and/or hypovolemic shock ( 2 cases), severe infection ( 2 cases), graft loss (returning to dialysis, retransplantation or death, 0 cases) and incomplete medical records ( 1 case) were excluded. There was no clinical evidence of acute allograft rejection. Finally,

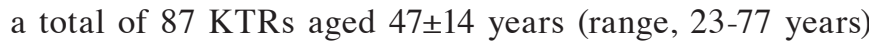
were enrolled in the present study, including 66 males and 21 females. They were divided into a CAD group $(n=42)$ and a non-CAD group $(n=45)$. The KTRs were treated with triple immunosuppressive regimens, including a glucocorticoid, mycophenolic acid and calcineurin inhibitor.

Diagnosis. The definition of CAD was based on the Kidney Disease Improving Global Outcomes guidelines, specifically an estimated glomerular filtration rate (eGFR) $<40 \mathrm{ml} / \mathrm{min}$ and/or proteinuria $>500 \mathrm{mg} /$ day (9). The Modification of Diet in Renal Disease Study formula was used to calculate the eGFR (10).

Observation indicators. The basic information of each patient was carefully recorded, including age, sex, weight, height, medical history (years after kidney transplantation, complications and infections), vital signs immediately after admission (temperature, heart rate and respiratory rate) and inflammatory markers, including white blood cells (WBC; reference range, $4-10 \times 10^{9}$ cells/l), neutrophil percentage $(\mathrm{N} \%$; reference range, 40-75\%), $\mathrm{PCT}$ and C-reactive protein (CRP; reference range, $0-10.0 \mathrm{mg} / \mathrm{l}$ ). A Sysmex XN9000 (Hyogo) was used for normal blood tests. A Cobas 8000 (Roche) was used to determine PCT with reference range of 0.021-0.500 ng/ml. An AU5800 (Beckman Coulter) was used to determine further biochemical indicators, including alanine aminotransferase (ALT; u/l), aspartate aminotransferase (AST; $\mathrm{u} / \mathrm{l})$, fasting blood glucose (FBG; $\mathrm{mmol} / \mathrm{l})$, creatinine $(\mu \mathrm{mol} / \mathrm{l})$, urea nitrogen $(\mathrm{mmol} / \mathrm{l})$, uric acid $(\mu \mathrm{mol} / \mathrm{l})$, triglyceride $(\mathrm{TG} ; \mathrm{mmol} / \mathrm{l})$, high-density lipoprotein (HDL; mmol/l), low-density lipoprotein (LDL; mmol/l) and lipoprotein(a) $[\mathrm{Lp}(\mathrm{a}), \mathrm{mg} / \mathrm{ll}$. Blood samples were measured within $24 \mathrm{~h}$ of admission and collected prior to the use of antibiotics.

Statistical analysis. SPSS 13.0 statistical software (SPSS, Inc.) was used for statistical analysis of all data. The Kolmogorov-Smirnov test was used to assess normality of distribution. Measurement data with a normal distribution are expressed as the mean \pm standard deviation. The median (first and third quartile) was used to represent measurement data with a non-normal distribution. Count data are expressed as $\mathrm{n}(\%)$. The independent samples t-test was used to compare data between two groups with a normal distribution. The Mann-Whitney U test was used to compare data between two groups with a non-normal distribution. The $\chi^{2}$-test was used for comparison of rates. The comparison between the diagnostic efficiencies of the different indicators was based on the receiver operating characteristic curve (ROC) analysis and area under the curve (AUC). The sensitivity and specificity were calculated and the cut-off value was determined using the Youden index. Univariate and multivariate binary logistic regression analysis was used to calculate odds ratios (ORs) with $95 \% \mathrm{CI}$ for the association of various parameters with the risk of $\mathrm{CAD}$. $\mathrm{P}<0.05$ was considered to indicate statistical significance.

\section{Results}

Clinical features of KTRs in the CAD and non-CAD group. The clinical features of KTRs in CAD and non-CAD group are listed in Table I. Compared with the non-CAD group, the CAD group had a higher ratio of male patients $(\mathrm{P}=0.010)$, a lower body mass index (BMI; $\mathrm{P}=0.041)$, a larger amount of years after transplantation $(\mathrm{P}<0.001)$, a higher serum concentration of PCT, N\% and $\mathrm{Lp}$ (a) ( $\mathrm{P}=0.001-0.004)$ and a lower serum concentration of AST, HDL and LDL ( $\mathrm{P}=0.001-0.044)$. No differences between the two groups were identified in terms of age, frequency of local infection, location of infection, comorbidities, CRP, WBC, ALT, FBG and TG (all P>0.05).

Comparison of inflammatory markers between infected and non-infected patients. Among the 87 KTRs, 44 cases (49.4\%) had an infection and 43 cases (50.6\%) were without infection. No difference between the infected and non-infected patients was identified in terms of PCT, WBC and N\% (all P>0.05), but the CRP in the infection group was significantly higher $(\mathrm{P}=0.045$; Table II).

ROC curves of potential biomarkers for predicting CAD in KTRs. ROC curve analysis indicated that the CRP and WBC were not able to predict CAD in KTRs ( $\mathrm{P}>0.05$; the $\mathrm{P}$-value is the probability that the observed sample AUC is found when, in fact, the true AUC is 0.5 ). The ability of PCT, N\%, AST, HDL, LDL and Lp(a) to predict CAD in KTRs was demonstrated in Table III. Among these, PCT had the largest AUC (0.893), with a sensitivity of $85.7 \%$ and a specificity of $90.0 \%$ at the best cut-off value $(0.086 \mathrm{ng} / \mathrm{ml})$, followed by $\mathrm{Lp}(\mathrm{a}), \mathrm{HDL}$ and LDL; N\% and AST had lower AUC (0.694 and 0.626, respectively) (Fig. 1).

Logistic regression analysis of CAD in patients after kidney transplantation. To determine the predictive value of PCT regarding $\mathrm{CAD}$ under exclusion of the interaction with other inflammatory indicators, logistic regression analysis was performed. The cohort of KTRs was stratified into two groups based on the serum PCT levels at the best cut-off value $(0.086 \mathrm{ng} / \mathrm{ml})$. CAD was regarded as the dependent variable, while gender, BMI, years after transplantation, PCT, N\% AST, HDL, LDL and Lp(a) were taken as independent variables (Table IV). The results indicated that elevated PCT was a unique independent risk factor for CAD in KTRs $(\mathrm{OR}=40.500$, 95\% CI=3.093-530.293, $\mathrm{P}=0.005$ ).

\section{Discussion}

Renal transplantation is considered a unique optimum therapy for patients with end-stage renal disease (ESRD). Despite the tremendous improvement in short-term renal allograft 
Table I. Clinical characteristics of KTRs in the CAD and non-CAD groups.

\begin{tabular}{|c|c|c|c|c|c|}
\hline Characteristic & Reference ranges & Non-CAD $(\mathrm{n}=45)$ & CAD $(n=42)$ & $\mathrm{t} / \chi^{2} / Z$ & P-value \\
\hline Age (years) & - & $44 \pm 16^{\mathrm{a}}$ & $50 \pm 10^{\mathrm{a}}$ & 1.888 & 0.063 \\
\hline Male gender & - & $29(64.4)^{\mathrm{b}}$ & $37(88.1)^{\mathrm{b}}$ & 6.636 & 0.010 \\
\hline BMI $\left(\mathrm{kg} / \mathrm{m}^{2}\right)$ & - & $21.99 \pm 3.27^{\mathrm{a}}$ & $20.50 \pm 3.35^{\mathrm{a}}$ & 2.076 & 0.041 \\
\hline Time after transplantation (years) & - & $6 \pm 5^{\mathrm{a}}$ & $11 \pm 5^{\mathrm{a}}$ & 3.847 & $<0.001$ \\
\hline Local infection & - & $22(48.9)^{\mathrm{b}}$ & $22(52.4)^{\mathrm{b}}$ & 0.106 & 0.745 \\
\hline \multicolumn{6}{|l|}{ Infection location } \\
\hline Respiratory tract & - & $10(22.2)^{\mathrm{b}}$ & $15(35.7)^{\mathrm{b}}$ & 1.931 & 0.165 \\
\hline Urinary tract & - & $8(17.8)^{b}$ & $4(9.5)^{b}$ & 0.647 & 0.421 \\
\hline Gastrointestinal tract & - & $4(8.9)^{b}$ & $4(9.5)^{b}$ & 0.000 & 0.786 \\
\hline \multicolumn{6}{|l|}{ Comorbidities } \\
\hline Diabetes mellitus & - & $2(4.4)^{b}$ & $8(19.0)^{b}$ & 3.232 & 0.072 \\
\hline Hypertension & - & $5(11.1)^{b}$ & $2(4.8)^{\mathrm{b}}$ & 0.481 & 0.488 \\
\hline Coronary heart disease & - & $0(0.0)^{\mathrm{b}}$ & $0(0.0)^{\mathrm{b}}$ & - & - \\
\hline Stroke & - & $0(0.0)^{\mathrm{b}}$ & $1(2.4)^{b}$ & - & 0.483 \\
\hline \multicolumn{6}{|l|}{ Laboratory parameters } \\
\hline PCT (ng/ml) & $0.021-0.500$ & $0.059(0.041,0.076)^{\mathrm{c}}$ & $0.250(0.163,0.644)^{\mathrm{c}}$ & 3.221 & 0.001 \\
\hline CRP (mg/l) & $0-10.0$ & $5.0(3.9,6.8)^{\mathrm{c}}$ & $6.1(4.4,8.0)^{\mathrm{c}}$ & 1.444 & 0.149 \\
\hline WBC $\left(\times 10^{9} / 1\right)$ & $4-10$ & $7.47(5.81,9.68)^{\mathrm{c}}$ & $6.79(5.33,8.66)^{\mathrm{c}}$ & 1.688 & 0.091 \\
\hline $\mathrm{N} \%$ & $40-75$ & $66.2(53.3,74.1)^{\mathrm{c}}$ & $74.8(68.0,80.9)^{\mathrm{c}}$ & 3.054 & 0.002 \\
\hline $\operatorname{ALT}(\mathrm{u} / \mathrm{l})$ & $9-50$ & $20(13,25)^{\mathrm{c}}$ & $13(7,24)^{\mathrm{c}}$ & 1.296 & 0.195 \\
\hline $\operatorname{AST}(\mathrm{u} / \mathrm{l})$ & $15-40$ & $17(15,21)^{\mathrm{c}}$ & $14(12,19)^{\mathrm{c}}$ & 2.010 & 0.044 \\
\hline $\mathrm{FBG}(\mathrm{mmol} / \mathrm{l})$ & $3.90-6.10$ & $4.51(4.00,5.08)^{\mathrm{c}}$ & $5.01(4.18,5.82)^{\mathrm{c}}$ & 1.939 & 0.052 \\
\hline $\mathrm{TG}(\mathrm{mmol} / \mathrm{l})$ & $0.70-2.02$ & $1.48(1.04,2.16)^{\mathrm{c}}$ & $1.30(0.89,2.30)^{\mathrm{c}}$ & 0.080 & 0.936 \\
\hline HDL (mmol/l) & $0.79-2.00$ & $1.40(1.05,1.53)^{\mathrm{c}}$ & $1.01(0.78,1.20)^{\mathrm{c}}$ & 4.132 & $<0.001$ \\
\hline LDL (mmol/l) & $1.50-3.36$ & $2.08(1.72,2.36)^{\mathrm{c}}$ & $1.57(1.18,1.91)^{\mathrm{c}}$ & 3.317 & 0.001 \\
\hline $\mathrm{Lp}(\mathrm{a})(\mathrm{mg} / \mathrm{l})$ & $0-300$ & $48(27,103)^{\mathrm{c}}$ & $121(73,239)^{\mathrm{c}}$ & 2.861 & 0.004 \\
\hline
\end{tabular}

${ }^{a}$ Values are expressed as the mean \pm standard deviation. ${ }^{b}$ Values are expressed as the $\mathrm{n}(\%) .{ }^{\mathrm{c}}$ Values are expressed as the median (first and third quartile). BMI, body mass index; PCT, procalcitonin; CRP, C-reactive protein; WBC, white blood cell count; N\%, neutrophil percentage; ALT, alanine aminotransferase; AST, aspartate aminotransferase; FBG, fasting blood glucose; TG, triglycerides; HDL, high-density lipoprotein; LDL, low-density lipoprotein; Lp(a), lipoprotein(a); CAD, chronic allograft dysfunction.

Table II. Comparison of inflammatory markers in the non-infection and local infection groups.

\begin{tabular}{|c|c|c|c|c|}
\hline Characteristic & Non-infection $(n=43)$ & Local infection $(n=44)$ & $\mathrm{Z}$ & P-value \\
\hline PCT (ng/ml) & $0.071(0.046,0.162)^{\mathrm{a}}$ & $0.187(0.058,0.419)^{\mathrm{a}}$ & 1.123 & 0.261 \\
\hline CRP (mg/l) & $4.9(3.9,6.6)^{\mathrm{a}}$ & $6.1(4.9,8.8)^{\mathrm{a}}$ & 2.008 & 0.045 \\
\hline WBC (x109/1) & $7.04(5.57,9.58)^{\mathrm{a}}$ & $7.18(5.35,9.11)^{\mathrm{a}}$ & 0.228 & 0.820 \\
\hline $\mathrm{N} \%$ & $68.7(58.1,75.3)^{\mathrm{a}}$ & $73.5(59.1,81.7)^{\mathrm{a}}$ & 1.749 & 0.080 \\
\hline
\end{tabular}

${ }^{\text {a}}$ Values are expressed as the median (first and third quartile). PCT, procalcitonin; CRP, C-reactive protein; WBC, white blood cell count; N\%, neutrophil percentage.

survival, long-term graft survival is limited, as most of KTRs fail within 10 years after transplantation. CAD is a complex process with multiple factors and is closely linked to progressive renal fibrosis and tubular atrophy.

CAD remains the leading cause of late graft failure (11). In the present study, the ratio of males in the CAD group was higher, which means that males may be more likely to have poor renal transplant outcome than females. The reasons may include more frequent acute rejection due to a higher likelihood of human leukocyte antigen mismatch, no advantage in hormones and complex immunological processes, as well as worse compliance to immunosuppressants (12). 
Table III. Predictive accuracy of various parameters for chronic allograft dysfunction in kidney transplant recipients determined by receiver operating characteristics curve analysis.

\begin{tabular}{lccccc}
\hline Parameter & AUC & P-value & Cut-off value & Sensitivity (\%) & Specificity (\%) \\
\hline PCT $(\mathrm{ng} / \mathrm{ml})$ & 0.893 & $<0.001$ & $>0.086$ & 85.7 & 90.0 \\
N\% & 0.694 & 0.001 & $>67.1$ & 80.0 & 54.5 \\
AST $(\mathrm{u} / \mathrm{l})$ & 0.626 & 0.045 & $\leq 15$ & 57.5 & 71.1 \\
HDL $(\mathrm{mmol} / \mathrm{l})$ & 0.767 & $<0.001$ & $\leq 1.27$ & 89.5 & 58.1 \\
LDL $(\mathrm{mmol} / \mathrm{l})$ & 0.715 & 0.001 & $\leq 1.82$ & 73.7 & 72.1 \\
Lp(a) $(\mathrm{mg} / \mathrm{l})$ & 0.770 & $<0.001$ & $>83$ & 72.7 & 70.6 \\
\hline
\end{tabular}

AUC, area under the curve; PCT, procalcitonin; N\%, neutrophil percentage; AST, aspartate aminotransferase; HDL, high-density lipoprotein; LDL, low-density lipoprotein; Lp(a), lipoprotein(a).



Figure 1. Receiver operating characteristic curves using PCT, HDL, LDL and $\mathrm{Lp}$ (a) to predict chronic allograft dysfunction of kidney transplant recipients. PCT exhibited the largest AUC compared with the other indexes. PCT, procalcitonin; HDL, high-density lipoprotein; LDL, low-density lipoprotein; Lp(a), lipoprotein(a); AUC, area under the curve.

Obesity and increased BMI are considered to be accompanied by an elevated incidence of renal allograft insufficiency or CAD $(13,14)$. However, the present results were not comparable with this, as the proportion of obese and overweight subjects in the CAD and the non-CAD group was very low. The lower BMI in the CAD vs. the non-CAD group of the present study may in part be explained by the distribution characteristics of the nutritional status of the subjects. Chronic inflammation, which is common in patients with uremia, is linked with malnutrition, resulting in a novel concept named malnutrition-inflammation complex syndrome, which was suggested to be independently associated with increased mortality risk and adverse transplant outcome (15).

The most noteworthy observation of the present study is the independent predictive value of serum PCT levels regarding the risk of progression to CAD in KTR patients. There are three reasonable explanations for this: i) The relatively high serum PCT level may reflect the activity of chronic low-grade inflammation in renal allograft rejection, which indirectly reflects the tendency of future progression to renal failure (16). Stimulated renal parenchymal cells may directly release PCT into the circulation in response to this sustained inflammatory response. Furthermore, indirectly, renal tissue also releases cytokines into the bloodstream, which induces an increase in chronic low-grade production and induces other tissues, possibly including adipose tissue, to release PCT into the circulatory pool $(17,18)$.ii) Proteinuria may be one of the mechanisms that link increased PCT concentration and CAD. van Ree et al (4) suggested that high levels of PCT, which may predict renal allograft failure, reflects the release of PCT by renal parenchymal cells into the circulation, and it is a feedback to the infiltration and activation of renal macrophages associated with proteinuria. iii) The same study indicated that PCT predicted non-proteinuric graft failure in KTRs, which may be due to the cascade of macrophage activity and interstitial inflammation being completely activated, even if the urinary protein level of renal tubular epithelial cells has not yet reached the upper limit of the reabsorption capacity, indicating PCT may be an earlier biomarker in the progression of CAD than proteinuria. Furthermore, the lower limit of the plasma PCT concentration for predicting the risk of CAD was $0.086 \mathrm{ng} / \mathrm{ml}$, which is well below that of the presence of infection detected with the method of the present study. Therefore, an ultrasensitive assay is required to detect the PCT concentration.

Chronic rejection specifically refers to the progressive loss function of the transplanted kidney, the pathogenesis of which shares identical pathways with atherosclerosis (19). $\mathrm{Lp}(\mathrm{a})$, similar to other biomarkers of lipid metabolism, is a genetically-determined and forceful risk factor for atherosclerosis contributing to cardiovascular events (CVEs) (20). Increased $\mathrm{Lp}$ (a) levels, also considered a prototype candidate for the uremic toxin, are present in patients with CKD, including those with ESRD on dialysis (21-23). In the present study, among all lipid analysis indicators, $\mathrm{Lp}$ (a) was the most effective in predicting CAD of KTRs, as the AUC was the largest. Therefore, $\mathrm{Lp}$ (a) may be superior to HDL and LDL in predicting CAD. However, the mechanism of lipid metabolism affecting the progress of CAD remains to be fully elucidated. There are two hypothesized mechanisms of action. The former mechanism is that of the pathogenesis of endothelial dysfunction and macrovascular and small-vessel disease, which is composed of pro-inflammatory and pro-thrombotic properties of $\mathrm{Lp}(\mathrm{a})$, and in addition, oxidized phospholipids may lead to inflammatory reactions in cells and tissues. The strongest data supporting the latter hypothesis come from a temporal 
Table IV. Univariate and multivariate logistic regression analysis of chronic allograft dysfunction in kidney transplant recipients.

\begin{tabular}{|c|c|c|c|c|}
\hline \multirow[b]{2}{*}{ Factor } & \multicolumn{2}{|c|}{ Univariate } & \multicolumn{2}{|c|}{ Multivariate } \\
\hline & OR $(95 \% \mathrm{CI})$ & P-value & OR $(95 \% \mathrm{CI})$ & P-value \\
\hline Age (years) & $1.030(0.998-1.063)$ & 0.069 & & \\
\hline Male gender & $4.083(1.338-12.459)$ & 0.013 & $1.030(0.060-17.788)$ & 0.984 \\
\hline BMI $\left(\mathrm{kg} / \mathrm{m}^{2}\right)$ & $0.871(0.761-0.997)$ & 0.045 & $1.006(0.661-1.531)$ & 0.978 \\
\hline Years after transplantation & $1.169(1.069-1.280)$ & 0.001 & $1.164(0.900-1.505)$ & 0.248 \\
\hline Diabetes mellitus & $5.059(1.008-25.396)$ & 0.049 & $2.074(0.383-11.226)$ & 0.397 \\
\hline $\operatorname{PCT}(>0.086 \mathrm{ng} / \mathrm{ml})^{\mathrm{a}}$ & $54.000(4.211-692.484)$ & 0.002 & $40.500(3.093-530.293)$ & 0.005 \\
\hline WBC (x109/1) & $0.863(0.725-1.027)$ & 0.096 & & \\
\hline $\mathrm{N} \%(>67.1)^{\mathrm{a}}$ & $4.800(1.809-12.737)$ & 0.002 & $0.175(0.002-16.428)$ & 0.452 \\
\hline $\operatorname{AST}(\leq 15 \mathrm{u} / 1)^{\mathrm{a}}$ & $3.330(1.355-8.185)$ & 0.009 & $2.205(0.065-74.753)$ & 0.660 \\
\hline $\operatorname{HDL}(\leq 1.27 \mathrm{mmol} / \mathrm{l})^{\mathrm{a}}$ & $11.806(3.555-39.203)$ & $<0.001$ & $7.467(0.201-276.716)$ & 0.275 \\
\hline $\operatorname{LDL}(\leq 1.82 \mathrm{mmol} / \mathrm{l})^{\mathrm{a}}$ & $7.233(2.708-19.322)$ & $<0.001$ & $7.594(0.317-181.925)$ & 0.211 \\
\hline $\mathrm{LP}(\mathrm{a})(>83 \mathrm{mg} / \mathrm{l})^{\mathrm{a}}$ & $6.400(1.573-26.034)$ & 0.010 & $9.364(0.312-281.445)$ & 0.198 \\
\hline
\end{tabular}

${ }^{a}$ Values in the brackets are the cutoff values of the parameters. BMI, body mass index; PCT, procalcitonin; WBC, white blood cell count; N\%, neutrophil percentage; AST, aspartate aminotransferase; HDL, high-density lipoprotein; LDL, low-density lipoprotein; Lp(a), lipoprotein(a); $\mathrm{OR}$, odds ratio.

evaluation of $\mathrm{Lp}$ (a) levels with a decline of $>35 \%$ in patients after renal transplantation (22). The latter mechanism is the decreased renal scavenging capability of $\mathrm{Lp}(\mathrm{a})$ in patients with renal failure, which has been demonstrated by the distribution difference of the plasma $\mathrm{Lp}$ (a) concentration in renal arteries and veins and by the urine apolipoprotein A fragments (22). Such a negative correlation between $\mathrm{Lp}$ (a) levels and renal function, triggering a vicious circle, leads to CVEs even in patients with early renal impairment $(22,24)$.

Based on a previous study, the predictive efficiency of PCT in local infection of CKD patients is not as good as that of traditional inflammatory biomarkers (e.g. CRP), yet it has a significant advantage in predicting sepsis (8). Similarly, in the present study, PCT was not able to identify local infection in renal transplant recipients. Thus, it may be speculated that in septic KTRs, PCT may principally participate in the systemic inflammatory response syndrome due to severe infection, while $\mathrm{Lp}$ (a) may be a more useful biomarker to predict CAD progression. Whether Lp(a) may be used as a valuable index for CAD in septic KTRs remains to be further explored.

Unlike previous studies, the present study did not exclude KTRs with focal infection, considering the high prevalence of infection due to the long-term use of immunosuppressive drugs. Of note, the levels of inflammatory markers, including PCT, N\% and WBC, exhibited no statistically significant difference between the infection and non-infection subgroups, suggesting that these inflammatory markers are not able to predict focal infection in KTRs. Combined with the results of a recent study (8), as a by-conclusion of the present study, it may be suggested that PCT cannot predict local infection in CKD patients and KTRs with or without CAD.

The present study had a few limitations: i) It was limited by its retrospective nature; ii) given the small sample size, the conclusions require further confirmation by a larger-sample survey; iii) the study was a single-center study with selection bias; iv) KTRs with severe infection, a common complication contributing to poor prognosis regarding graft survival, were not included in the study; v) the mechanism of lipid metabolism affecting the progress of CAD remains elusive.

In conclusion, $\mathrm{PCT}$ was proved to be a unique independent risk factor of CAD in KTRs. However, PCT, WBC and N\% cannot predict local infection in KTRs except CRP. Therefore, even in KTRs with local infection, elevated PCT may still be a potential indicator for poor prognosis regarding renal graft survival.

\section{Acknowledgements}

Not applicable.

\section{Funding}

The present study was funded by the Fifth-phase Research Project Funding Program for the '333 Project' in Jiangsu Province (grant no. BRA 2017116), the Science and Technology Bureau Supporting Project in Changzhou City (grant no. CE 20175030) and Health Top-notch Personnel in Changzhou City (grant no. KY 201757).

\section{Availability of data and materials}

The datasets used and/or analyzed during the current study are available from the corresponding author on reasonable request.

\section{Authors' contributions}

JY and YS conceived of the study, and drafted the manuscript. LJ and DX carried out the experiments. YS participated in the 
design of the study and performed the statistical analysis. All authors read and approved the final manuscript.

\section{Ethics approval and consent to participate}

The present study was approved by the Ethics Committee of the Third Affiliated Hospital of Soochow University (Changzhou, China) and all patients signed informed consent forms.

\section{Patient consent for publication}

Not applicable.

\section{Competing interests}

The authors declare that they have no competing interests.

\section{References}

1. Yu XY, Wang Y, Zhong H, Dou QL, Song YL and Wen H: Diagnostic value of serum procalcitonin in solid organ transplant recipients: A systematic review and meta-analysis. Transplant Proc 46: 26-32, 2014.

2. Bhatti $\mathrm{AB}$ and Usman $\mathrm{M}$ : Chronic renal transplant rejection and possible anti-proliferative drug targets. Cureus 7: e376, 2015.

3. Kreis HA and Ponticelli C: Causes of late renal allograft loss: Chronic allograft dysfunction, death, and other factors Transplantation 71 (Suppl 11): SS5-SS9, 2001.

4. van Ree RM, de Vries AP, Oterdoom LH, Seelen MA, Gansevoort RT, Schouten JP, Struck J, Navis G, Gans RO, van der Heide JJ, et al: Plasma procalcitonin is an independent predictor of graft failure late after renal transplantation. Transplantation 88: 279-287, 2009.

5. Sager R, Kutz A, Mueller B and Schuetz P: Procalcitonin-guided diagnosis and antibiotic stewardship revisited. BMC Med 15: 15, 2017.

6. Dumea R, Siriopol D, Hogas S, Mititiuc I and Covic A: Procalcitonin: Diagnostic value in systemic infections in chronic kidney disease or renal transplant patients. Int Urol Nephrol 46 461-468, 2014

7. Grace E and Turner RM: Use of procalcitonin in patients with various degrees of chronic kidney disease including renal replacement therapy. Clin Infect Dis 59: 1761-1767, 2014.

8. Sun Y, Jiang L and Shao X: Predictive value of procalcitonin for diagnosis of infections in patients with chronic kidney disease: A comparison with traditional inflammatory markers C-reactive protein, white blood cell count, and neutrophil percentage. Int Urol Nephrol 49: 2205-2216, 2017.

9. Kidney Disease: Improving Global Outcomes (KDIGO) Transplant Work Group: KDIGO clinical practice guideline for the care of kidney transplant recipients. Am J Transplant 9 (Suppl 3): S1-S155, 2009.
10. National Kidney Foundation: K/DOQI clinical practice guidelines for chronic kidney disease: Evaluation, classification, and stratification. Am J kidney Dis 39 (2 Suppl 1): S1-S266, 2002.

11. Kaplan B: Overcoming barriers to long-term graft survival. Am J Kidney Dis 47 (4 Suppl 2): S52-S64, 2006.

12. Chen PD, Tsai MK, Lee CY, Yang CY, Hu RH, Lee PH and Lai HS: Gender differences in renal transplant graft survival. J Formos Med Assoc 112: 783-788, 2013.

13. Grosso G, Corona D, Mistretta A, Zerbo D, Sinagra N Giaquinta A, Caglià $\mathrm{P}$, Amodeo $\mathrm{C}$, Leonardi $\mathrm{A}$, Gula $\mathrm{R}$, et al: The role of obesity in kidney transplantation outcome. Transplant Proc 44: 1864-1868, 2012.

14. Papalia T, Greco R, Lofaro D, Maestripieri S, Mancuso D and Bonofiglio R: Impact of body mass index on graft loss in normal and overweight patients: Retrospective analysis of 206 renal transplants. Clin Transplant 24: E241-E246, 2010.

15. Molnar MZ, Czira ME, Rudas A, Ujszaszi A, Lindner A, Fornadi K, Kiss I, Remport A, Novak M, Kennedy SH, et al: Association of the malnutrition-inflammation score with clinical outcomes in kidney transplant recipients. Am J Kidney Dis 58: 101-108, 2011.

16. Vazquez MA, Jeyarajah DR, Kielar ML and Lu CY: Long-term outcomes of renal transplantation: A result of the original endowment of the donor kidney and the inflammatory response to both alloantigens and injury. Curr Opin Nephrol Hypertens 9: 643-648, 2000.

17. Becker KL, Nylén ES, White JC, Müller B and Snider RH Jr: Clinical review 167: Procalcitonin and the calcitonin gene family of peptides in inflammation, infection, and sepsis: A journey from calcitonin back to its precursors. J Clin Endocrinol Metab 89: 1512-1525, 2004

18. Becker KL, Snider R and Nylen ES: Procalcitonin assay in systemic inflammation, infection, and sepsis: Clinical utility and limitations. Crit Care Med 36: 941-952, 2008.

19. Wahn F, Daniel V, Kronenberg F, Opelz G, Michalk DV and Querfeld U: Impact of apolipoprotein(a) phenotypes on long-term renal transplant survival. J Am Soc Nephrol 12: 1052-1058, 2001.

20. Kronenberg F: Human genetics and the causal role of lipoprotein(a) for various diseases. Cardiovasc Drugs Ther 30: 87-100, 2016.

21. Florens N, Calzada C, Lyasko E, Juillard L and Soulage CO: Modified lipids and lipoproteins in chronic kidney disease: A new class of uremic toxins. Toxins (Basel) 8: E376, 2016.

22. Kronenberg F: Causes and consequences of lipoprotein(a) abnormalities in kidney disease. Clin Exp Nephrol 18: 234-237, 2014.

23. Barter P: Lipoprotein metabolism and CKD: Overview. Clin Exp Nephrol 18: 243-246, 2014

24. Catena C, Colussi G, Nait F, Pezzutto F, Martinis F and Sechi LA: Early renal failure as a cardiovascular disease: Focus on lipoprotein(a) and prothrombotic state. World J Nephrol 4: 374-378, 2015.

This work is licensed under a Creative Commons Attribution-NonCommercial-NoDerivatives 4.0 International (CC BY-NC-ND 4.0) License. 